\title{
DELAMINATION OF POLYIMIDE IN HYDROFLUORIC ACID
}

\author{
Asaad K. EdaAn Al-mashaal ${ }^{a, *}$, Rebecca Cheung ${ }^{b}$ \\ ${ }^{a}$ University of Basrah, Polymer Research Centre, Material Science Department, Basrah, 61004, Iraq \\ ${ }^{b}$ The University of Edinburgh, Scottish Microelectronics Centre, School of Engineering, Edinburgh EH9 3FF, UK \\ * corresponding author: asaad.edaan@uobasrah.edu.iq
}

\begin{abstract}
Wet etching is a critical fabrication step for the mass production of micro and nanoelectronic devices. However, when an extremely corrosive acid such as hydrofluoric (HF) acid are used during etching, an undesirable damage might occur if the device includes a material that is not compatible with the acid. Polyimide thin films can serve as sacrificial/structural layers to fabricate freestanding or flexible devices. The importance of polyimide in microelectronics is due to its relatively low stress and compatibility with standard micromachining processes. In this work, a fast delamination process of a $4-\mu \mathrm{m}$-thin film of polyimide from a silicon substrate has been demonstrated. The films' detachment has been performed using a wet-based etchant of HF acid. Specifically, the effect of HF concentration on the delamination time required to detach the polyimide film from the substrate has been investigated. This study is intended to provide the information on the compatibility of using polyimide films with $\mathrm{HF}$, which can help in the design and fabrication of polyimide-based devices.
\end{abstract}

KEYWorDs: Polyimide, delamination, wet etching, hydrofluoric acid.

\section{INTRODUCTION}

The fabrication of microelectronic devices and their subsets involves the use of a range of acids, organic/inorganic polymers, metals, and semiconductor materials. The fabrication process includes several steps, such as deposition, photolithography, and etching. For example, it is common in the fabrication of microelectromechanical systems (MEMS) that a sacrificial layer of a material of interest is deposited on a substrate before it is subsequently removed by a specific type of etchants. However, there might be a considerable reliability concern when the materials/processes used in the fabrication are not compatible with each other [1]. The incompatibility could result from fabrication processes that require the use of chemicals and materials with different properties [2]. In this work, we focus on determining whether the application of a layer of polyimide is fully compatible with the etching process when a wet-based etchant of hydrofluoric (HF) acid is used during the fabrication process.

Polymers play a crucial role as essential functional and structural materials in modern micromachining technologies 3 . One of the polymeric materials that have found their way to several applications, especially in microelectronics and MEMS devices, is polyimide. The importance of polyimide films is due to their outstanding thermal, mechanical, and chemical properties. For example, the low modulus, high mechanical strength and high thermal stability have enabled the polyimide films to be used as sacrificial layers in suspended MEMS-based structures [4,5]. Another important application of polyimide-based layers is their use as active structural components in MEMS sensors and resonators $[6]$. In addition to their main func- tion as dielectric, alignment, passivation and insulator layers 9, polyimides have other useful applications such as a flexible substrate for different types of soft devices [10 12].

Hydrofluoric acid (HF) is one of the most widely used etchants in manufacturing industries. Despite the fact that etching in HF is a wet process, which may have a number of drawbacks, it is still considered as a highly preferred etching process because of the high etch rate and compatibility with batch fabrication processes [13, 14]. However, there seems to be a delamination issue with the use of HF solution when a layer of polyimide is attached to a silicon substrate. The delamination of the polyimide film occurs when the reacting ions of the HF solution are diffused into the interfacial area between the polyimide film and the substrate. Although a number of studies have been reported on both wet [15, 16] and dry [17,19] etching of polyimides, the delamination of polyimide films, especially in HF-based etchant solutions, has not been investigated yet. The purpose of the current paper is to demonstrate the detachment of a thin film of polyimide from a rigid silicon substrate. Particularly, we have investigated how the concentration of the HF etchant affects the delamination time and etching rate of polyimide films. The main motivation for conducting this work is to explore the possibility of developing a fast release process that can be utilised to detach a layer of polyimide from a silicon substrate without damaging the released layer or the substrate underneath. This is important as it can help in realising polyimide-based electronic devices. It is worth pointing out that the delamination process was conducted in a cleanroom environment (i. e., room temperature and atmospheric pressure). 


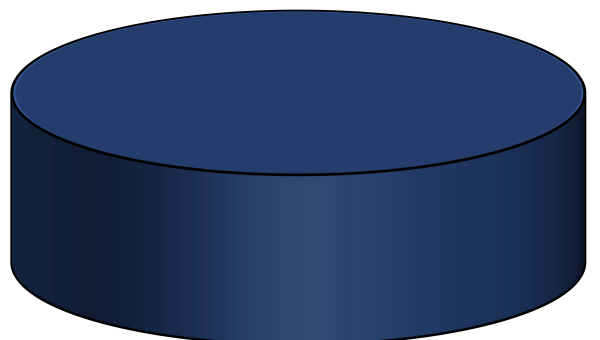

(A).

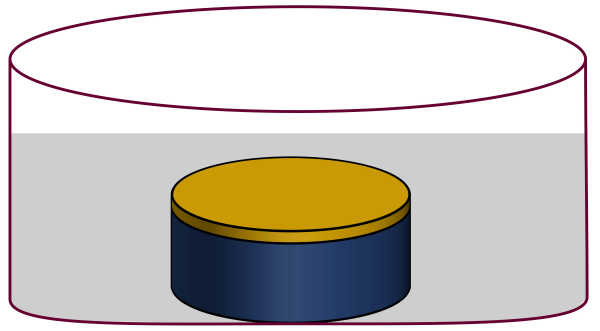

(C).

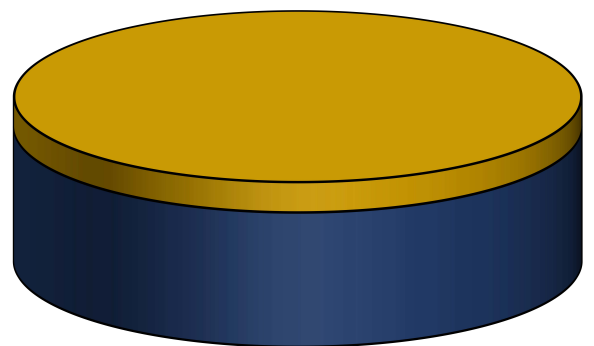

(B).

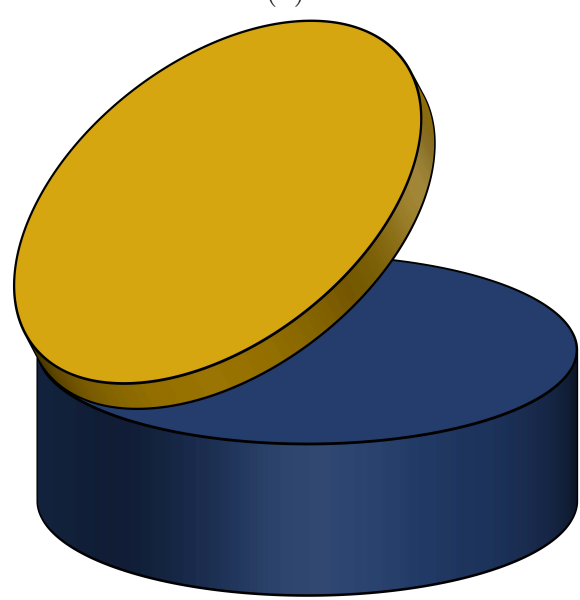

(D).

FIGURE 1. Fabrication and delamination processes for polyimide films. (A) Silicon substrate cleaned and primed with a promoter of VM-652. (B) Polyimide spin coated on silicon substrate and fully cured. (C) Substrate with polyimide on top immersed in HF-based acid. (D) Polyimide film delaminated from the substrate.

The chemical identity/composition of polyimide (PI 2611-HDMicroSystems) used in the present study is N-Methyl-2-pyrrolidone (85-95\%) and polyimide (10$20 \%)$.

\section{EXPERIMENTAL}

A schematic diagram of the deposition and delamination processes of polyimide films is shown in Figure 1. Firstly, a substrate of a p-type silicon wafer has been cleaned in acetone and isopropyl alcohol (IPA) solvents and treated with oxygen plasma (Barrel Asher) before being coated with a promoter of VM-652 (HDMicroSystems) (Figure 1a). The purpose of adding the promoter is to provide adhesion between the polymeric film to be applied and the silicon substrate. Then, a thin layer of polyimide (PI 2611-HDMicroSystems) has been spin-coated at $4000 \mathrm{rpm}$ for 45 seconds (Figure 1b). An initial bake at $110^{\circ} \mathrm{C}$ for 90 seconds has been performed. In order to cure the polyimide film completely, the sample has been held in an oven at $200{ }^{\circ} \mathrm{C}$ for 60 minutes. The thickness of the applied polyimide film has been measured to be $\sim 4 \mu \mathrm{m}$. After being immersed in the HF-based solution (Figure 1c), the polyimide film has been detached from the substrate (Figure 1d). It should be noted that due to the corrosive nature of $\mathrm{HF}$, it must be handled with extreme caution.

\section{Results AND Discussion}

The delamination process of polyimide films has been performed under the use of $\mathrm{HF}$ as the main etchant material. The three etchants include HF (49\%), 7:1 buffered hydrofluoric acid (BHF) and HF (49\%): deionised water (DI) with a ratio of $1: 1$. These proportions have been chosen as they are the most common in HF-based etching. The delamination procedure involves the immersion of the silicon/film stack in the prepared etchants until the entire polyimide film is delaminated. After submerging the silicon wafer that bears the polyimide film in the prepared solution, the film delamination has been inspected visually using a conventional camera. In such an inspection process, we have monitored how the film starts to delaminate at the edge and ultimately how the entire film is detached completely from the substrate. Both the edge and the full delamination time of polyimide films has been calculated and presented in Figure 2 Particularly, the approximate time required to delaminate the polyimide film from the substrate from the moment the entire silicon/film system was immersed in the etchant solution has been determined.

In general, the reason that the delamination start from the film's edge region is possibly associated with the shear stresses that are concentrated near the edges and film-substrate interfaces [20. Figure 2a shows a prepared sample being submerged in the HF solution. As HF molecules penetrate the interfacial area at the 


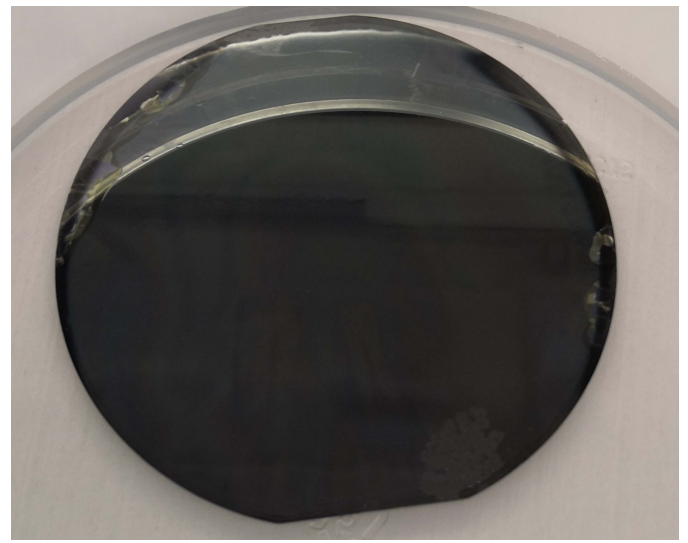

(A).

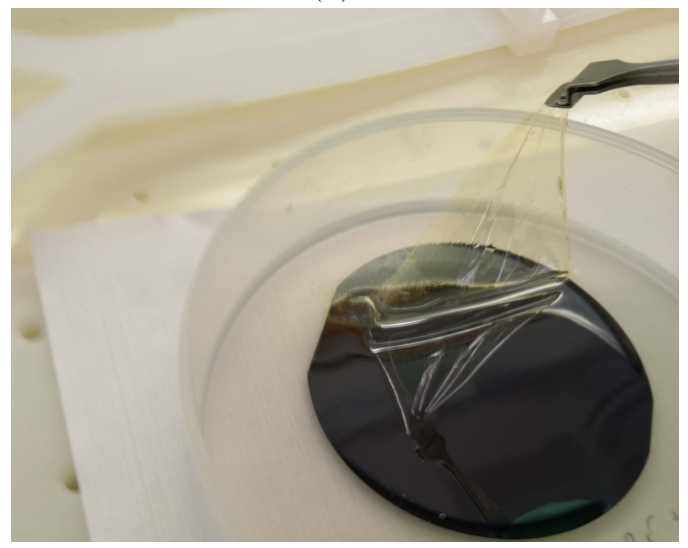

(c).

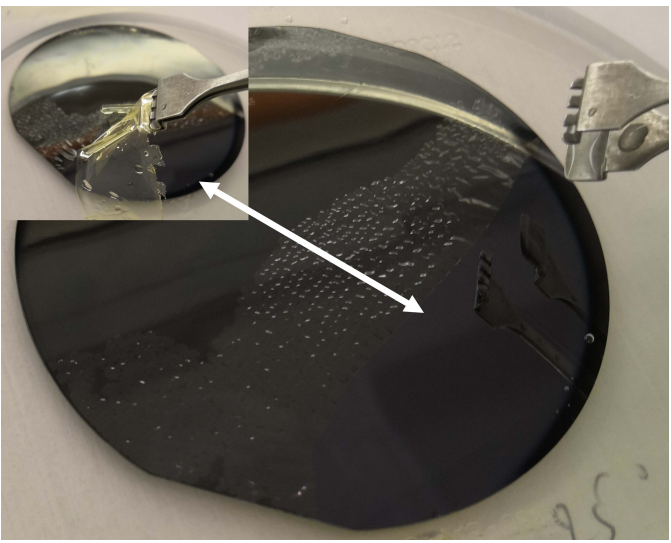

(в).

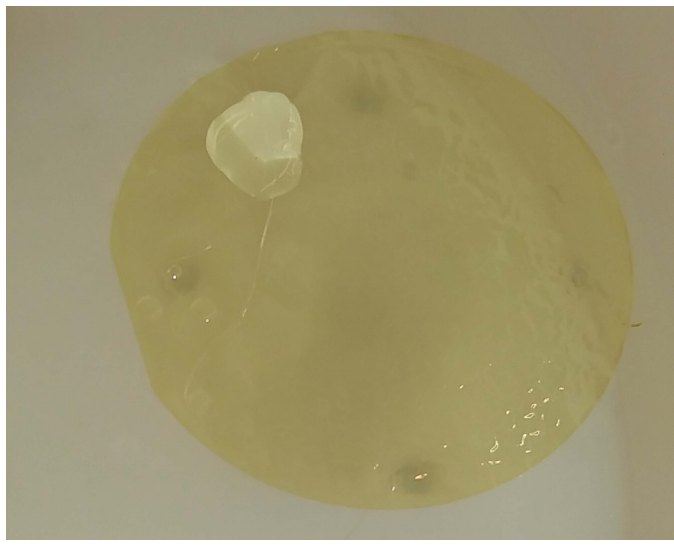

(D).

Figure 2. Delamination process of polyimide films in HF-based etchants. (A) Silicon substrate with the film on top is dipped in HF. (B) The film being delaminated around the edge of the substrate with the inset shows the film peeled off (C) A full detachment of the film from the substrate. (D) A delaminated polyimide film floating on deionised water subsequent to 4 minutes of immersion in HF.

rim of the wafer, the polyimide film appears to lose its adhesion to the silicon substrate at these far edge regions (Figure 2b). We believe that the promoter could play a role in the delamination process since it can be attacked by HF. It has been found that the size of delaminated regions is much smaller when an adhesion promoter is applied [21]. The inset in Figure $2 \mathrm{~b}$ shows how the edge-detached polyimide film has been peeled off from the silicon substrate. Once the etchant molecules penetrate deeply into the middle area and reach the entire interfacial region, the film can be easily delaminated as shown in Figure 2c. The final delaminated film can be seen floating on the DI water surface (Figure 2d).

Figure 3 shows the delamination time estimated for the polyimide film with respect to the three different types of HF-based etchants. For the first etchant solution, $50 \mathrm{ml}$ of $\mathrm{HF}$ ( $49 \%$ ) has been prepared. The edge delamination has been found to occur after $\sim 90$ seconds of immersion. The time required to attain a full delamination has been found to be approximately 4 minutes. In the case of the second etchant, a mixture of $50 \mathrm{ml}$ of $49 \% \mathrm{HF}$ and $50 \mathrm{ml}$ of DI water has been prepared. It appears that the edge and full delamination of polyimide films immersed in
HF: DI solution occurs after approximately 4 and 7 minutes, respectively (see Figure 3). Compared to the first etchant, the polyimide delamination time in HF: DI etchant has been observed to be about two times longer.

To prepare the third etchant of buffered hydrofluoric acid (BHF) solution, 7 parts of a buffering agent of ammonium fluoride $\left(\mathrm{NH}_{4} \mathrm{~F}\right)$ have been mixed with 1 part of HF (49\%). As shown in Figure 3 the time required for the polyimide film to detach from the substrate has been calculated to be $\sim 15$ minutes in the edge region. To achieve a complete detachment, however, the film must be immersed in the solution for more than 20 minutes. It is clear that the delamination of polyimide films is relatively slower in the BHF than that in the concentrated HF and HF: DI.

To inspect the etch rate of polyimide, the films' thickness has been measured using a reflectometer (Nanometrics Nanospec) before and after the immersion in the etchants. It has been found that the polyimide in the HF is around $15-25 \mathrm{~nm} / \mathrm{min}$. Compared to the total thickness of the as-applied film (e.g., $4 \mu \mathrm{m})$, the proportion of the layer that has been etched away in HF during the delamination does not exceed $5 \%$. No considerable difference has been measured in 


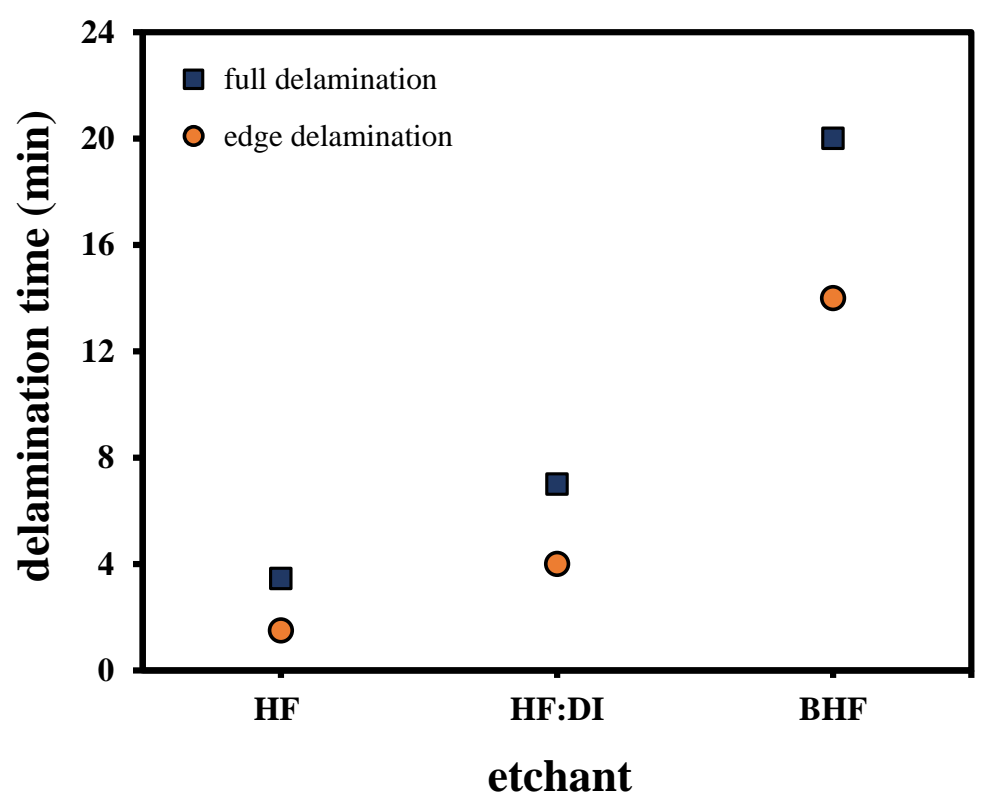

FiguRE 3. Delamination time of polyimide films as a function of different types of HF-based etchants.

the films' thickness after submerging the films in the HD: DI and BHF etchants. Such etching rates are for fully cured polyimide films, and they are in agreement with other reported findings [15]. It is worth pointing out that the etching rate of deposited or spin-coated (i. e., not cured) polyimide films is higher than that of the fully cured films.

The mechanism behind the delamination of thin polymeric films from a solid substrate is related to several possible effects. For example, it could result from the interfacial deformation process of the applied film and the molecular reaction of the etchant solution (liquid) and the silicon substrate (solid) 22. An alternative possible mechanism is that the HF attacks the interface, which causes the edge to peel up and delaminate. The residual induced stresses will cause the film to lift and continuously expose the whole interface to the attack of the HF. Under such a cascading effect, a full delamination of the film can be achieved. The HF has the ability to penetrate through the polyimide layer and attack the interface. Since our delamination process is HF dependent, the $\mathrm{HF}$ molecules are absorbed into the polyimide film and cause it to swell. As the HF is being absorbed, it is going to migrate to the polyimide/Si interface and consequently attack it. In general, we believe that both mechanisms could play a role. Compared to other reported techniques 2325 , the delamination of polyimide films in HF-based solutions that has been implemented in this work can be considered fast, simple and effective. Our simple method can be further improved to pave the way for a large-scale production of polyimide thin films for a wide range of flexible devices.

\section{Conclusion}

We have demonstrated the delamination of thin polyimide films in a wet-based etchant. Polyimide films of a thickness of around $4 \mu \mathrm{m}$ attached (spin coated) to a silicon substrate have been immersed in HF-based etchant solutions to investigate how the etchants can affect the detachment of the polymeric films from the substrate. It has been found that the purer (not fully diluted) the etchant solution, the greater the delamination of the film. A full delamination of the polyimide films occurs after $\sim 4,7$ and 15 minutes of immersion in HF (49\%), HF: DI and 7:1 BHF, respectively. The relatively long delamination time can be compensated by taking into account the least harmful etchant to the deposited film and the substrate. The results obtained in this work provide useful information on the compatibility between polyimide films and wet etching processes when HF-based etchants are involved.

\section{REFERENCES}

[1] J. Iannacci. Reliability of MEMS: A perspective on failure mechanisms, improvement solutions and best practices at development level. Displays 37:62-71, 2015. https://doi.org/10.1016/j.displa.2014.08.003.

[2] E. H. Cook, D. J. D. Carter. MEMS process compatibility of multiwall carbon nanotubes. Journal of Vacuum Science \& Technology B 29(6):06FE04, 2011. https://doi.org/10.1116/1.3662082

[3] B. J. Kim, E. Meng. Review of polymer MEMS micromachining. Journal of Micromechanics and Microengineering 15(1):013001, 2015. https://doi.org/10.1088/0960-1317/26/1/013001.

[4] A. K. Al-mashaal, G. Wood, E. Mastropaolo, R. Cheung. Electrostatically driven long-microbeams for low-frequency applications. Electronics Letters 54(6):372374, 2018. https://doi.org/10.1049/el.2017.4534. 
[5] A. Al-mashaal, E. Mastropaolo, A. Bunting, et al. Fabrication and characterisation of suspended microstructures of tantalum. Journal of Micromechanics and Microengineering 27(1):015020, 2017. https://doi.org/10.1088/0960-1317/27/1/015020

[6] J. Liu, Z. Shu, C. Wang, et al. Fabrication of a flexible polyimide-based electrostatically actuated MEMS relay. Journal of Micromechanics and Microengineering 28(10):105004, 2018. https://doi.org/10.1088/1361-6439/aacd2b.

[7] K. S. Choi, D. S. Kim, H. J. Yang, et al. A highly sensitive humidity sensor with a novel hole array structure using a polyimide sensing layer. $R S C$ Advances 4(61):32075-32080, 2014. https://doi.org/10.1039/c4ra02692f

[8] G. Zhang, V. Chu, J. P. Conde. Electrostatically actuated bilayer polyimide-based microresonators. Journal of Micromechanics and Microengineering 17(4):797-803, 2007. https://doi.org/10.1088/0960-1317/17/4/017

[9] Q.-H. Lu, F. Zheng. Polyimides for electronic applications. In Advanced Polyimide Materials, chap. 5, pp. 195-255. Elsevier, 2018. https: //doi.org/10.1016/b978-0-12-812640-0.00005-6.

[10] M. Gunda, P. Kumar, M. Katiyar. Review of mechanical characterization techniques for thin films used in flexible electronics. Critical Reviews in Solid State and Materials Sciences 42(2):129-152, 2017. https://doi.org/10.1080/10408436.2016.1186006

[11] S. Y. Xiao, L. F. Che, X. X. Li, Y. L. Wang. A novel fabrication process of MEMS devices on polyimide flexible substrates. Microelectronic Engineering 85(2):452-457, 2008. https://doi.org/10.1016/j.mee.2007.08.004

[12] C. Liu. Recent developments in polymer MEMS. Advanced Materials 19(22):3783-3790, 2007. https://doi.org/10.1002/adma.200701709

[13] D. Zhuang, J. H. Edgar. Wet etching of GaN, AlN, and SiC: a review. Materials Science and Engineering: $R$ : Reports 48(1):1-46, 2005. https://doi.org/10.1016/j.mser.2004.11.002

[14] D. M. Knotter. Etching mechanism of vitreous silicon dioxide in HF-based solutions. Journal of the American Chemical Society 122(18):4345-4351, 2000. https://doi.org/10.1021/ja993803z

[15] K. R. Williams, K. Gupta, M. Wasilik. Etch rates for micromachining processing - Part II. Journal of Microelectromechanical Systems 12(6):761-778, 2003. https://doi.org/10.1109/JMEMS . 2003.820936
[16] H. Ji-song, T. Zhi-yong, K. Sato, M. Shikida. Polyimide film micromachining by wet-etching technology. IEEJ Transactions on Sensors and Micromachines 125(1):27-36, 2005. https://doi.org/10.1541/ieejsmas.125.27

[17] A. Zulfiqar, A. Pfreundt, W. E. Svendsen, M. Dimaki. Fabrication of polyimide based microfluidic channels for biosensor devices. Journal of Micromechanics and Microengineering 25(3):035022, 2015. https://doi.org/10.1088/0960-1317/25/3/035022

[18] T. N. T. Nguyen, N. E. Lee. Deep reactive ion etching of polyimide for microfluidic applications. Journal of the Korean Physical Society 51(3):984-988, 2007. https://doi.org/10.3938/jkps.51.984

[19] U. Buder, J. P. von Klitzing, E. Obermeier. Reactive ion etching for bulk structuring of polyimide. Sensors and Actuators A: Physical 132(1):393-399, 2006. https://doi.org/10.1016/j.sna.2006.04.048.

[20] K. L. Mittal (ed.). Adhesion Measurement of Films and Coatings. CRC Press, 2014. https://doi.org/10.1201/b12020

[21] B. Erdem Alaca, M. T. A. Saif, H. Sehitoglu. On the interface debond at the edge of a thin film on a thick substrate. Acta Materialia 50(5):1197-1209, 2002. https://doi.org/10.1016/S1359-6454(01)00421-9

[22] D. S. Wie, Y. Zhang, M. K. Kim, et al. Wafer-recyclable, environment-friendly transfer printing for large-scale thin-film nanoelectronics. Proceedings ot the National Academy of Sciences of the United States of America 115(31):E7236-E7244, 2018. https://doi.org/10.1073/pnas.1806640115

[23] M. W. Czabaj, A. T. Zehnder, C. Y. Hui. Delamination of moisture saturated graphite/polyimide composites due to rapid heating. Composites Part B: Engineering 41(7):568-577, 2010. https: //doi.org/10.1016/j.compositesb.2010.05.008.

[24] J. Y. Oh, D. S. Park, B. S. Shin. Surface delamination of polyimide using 355-nm nanosecond pulse laser. Applied Physics B 113:411-415, 2013. https://doi.org/10.1007/s00340-013-5478-2.

[25] T. Walter, M. Lederer, G. Khatibi. Delamination of polyimide/Cu films under mixed mode loading. Microelectronics Reliability 64:281-286, 2016. https://doi.org/10.1016/j.microrel.2016.07.100 